\title{
IMPACTO DE LA VARIABILIDAD CLIMÁTICA SOBRE LA RECARGA A LOS ACUÍFEROS EN LA CUENCA DEL RÍO POÁS, VALLE CENTRAL, COSTA RICA
}

\author{
Asdrúbal Vargas ${ }^{1} \&$ Teresita Aguilar ${ }^{2}$ \\ ${ }^{1}$ C.S. Exploración Subterránea, Instituto Costarricense de Electricidad \\ 2 Escuela de Geología, Universidad de Costa Rica \\ E-mail: avargass@ice.go.cr / aaguilar@geologia.ucr.ac.cr
}

\begin{abstract}
We analyzed the impact of climate variation over the distribution of the hydric resources in río Poás watershed. Monthly hydric balances were carried out through the period 1976-2001. It is possible to identify humid subperiods and a dry one, lasting each of them about 7 years. During the dry subperiod, annual recharge reaches $1000 \mathrm{~mm}$.

In 1986 it was recorded $2019 \mathrm{~mm}$ rainfall, the lowest in the watershed, which is $22 \%$ less than the average; potential recharge was 36\% lower $(850 \mathrm{~mm})$, and runoff, $32 \%$ lower. The highest rainfall was recorded in 1996, with $3646 \mathrm{~mm}, 40 \%$ higher than the average, potential recharge was 65\% higher (2205 mm), and runoff, 30\% higher than the average ones. It is considered that between 24 and $48 \%$ of precipitation becomes potential recharge and most of it occurs during September and October.

There are stratigraphic and paleontological evidences that demonstrate climatic changes in the past. Those changes altered precipitation and temperature conditions, and therefore the distribution of water resources, without any anthrropic influence.
\end{abstract}

\begin{abstract}
RESUMEN: Se analiza el impacto de la variabilidad climática sobre la distribución del recurso hídrico en la cuenca del río Poás y se llevaron a cabo balances hídricos mensuales en el período comprendido entre 1976 a 2001. Se identifican subperíodos húmedos y un subperíodo seco, de alrededor de 7 años de duración cada uno. Durante el período seco la recarga anual alcanza valores de $1000 \mathrm{~mm}$.

En el año 1986 se registró la menor precipitación en la cuenca alcanzando 2019 mm, lo que significó un $22 \%$ menos que el promedio. La mayor precipitación se registró en el año de 1996 con 3646 mm, es decir un $40 \%$ mayor que el promedio. Para el año 1986 se obtuvo una recarga potencial de $850 \mathrm{~mm}$, un $36 \%$ menor que el promedio y para 1996 se obtuvo una recarga de $2205 \mathrm{~mm}$ es decir un $65 \%$ mayor que el promedio. Además, la escorrentía fue en 1986 un $32 \%$ menor y en 1996 un $30 \%$ mayor que el promedio respectivamente. El porcentaje de la precipitación que se considera como recarga potencial media anual varía de $24 \%$ hasta $48 \%$ y gran parte de ella ocurre en los meses de setiembre y octubre.

Existen evidencias estratigráficas y paleontológicas que demuestran en el pasado cambios climáticos que han alterado las condiciones de precipitación y temperatura y consecuentemente de la distribución del agua sin la influencia directa del ser humano.
\end{abstract}

\section{INTRODUCCIÓN}

Las investigaciones sobre el cambio climático y sus posibles efectos en el ciclo hidrológico, la biodiversidad y las actividades humanas, son relativamente recientes y se han llevado a cabo en mayor número en Europa o en Norteamérica. En 1988 el panel intergubernamental sobre cambio climático (IPCC), bajo el auspicio de la Organización Meteorológica Mundial, publicó 
un informe que indica la magnitud del cambio climático global. Las investigaciones del IPCC sugieren que la tendencia presente podría incrementar la temperatura media global en cerca de $1^{\circ} \mathrm{C}$ para el 2005 y $3^{\circ} \mathrm{C}$ antes del final del siglo XXI. Además, en la segunda conferencia sobre clima global se señaló que los impactos más importantes del cambio climático podrían ser sus efectos sobre el ciclo hidrológico (ECLAC, 1993). A nivel nacional, varios informes se refieren a la disponibilidad del agua y los posibles efectos del cambio climático en Costa Rica. MINAE (2000) presentó la primera comunicación nacional de Costa Rica ante la Convención Marco de las Naciones Unidas sobre Cambio Climático. En dicha comunicación se señala los escenarios y posibles áreas geográficas que tendrían una reducción de la lluvia y consecuentemente de la recarga a los acuíferos. El Observatorio del Desarrollo (2002), basado en diversos autores, presenta consideraciones sobre la disponibilidad del recurso hídrico y las presiones sobre la disponibilidad del recurso agua e intenta establecer estadísticas sobre los factores que rodean el recurso hídrico. Por otro lado, producto de un proyecto de investigación que realizaron la Oficina Regional para América Latina y la Escuela de Estudios Forestales y Ambientales de la Universidad de Yale en Estados Unidos se dispone de una publicación denominada Cambio Climático y Desarrollo, donde entre sus artículos sobresale el que plantea soluciones para mitigar los impactos del cambio climático por medio de un desarrollo sostenible, proponiendo un aumento de la eficacia energética, la adopción de fuentes de energía renovables y el uso más limpio de los combustibles convencionales (Johansson \& Karlsson en PNUMA \& WMO, 2000).

El programa de las Naciones Unidas para el Desarrollo (PNUD) apoya proyectos de investigación relacionados con el inminente cambio climático. Por lo tanto, un grupo de investigadores de la Escuela Centroamericana de Geología de la Universidad de Costa Rica y del Instituto Costarricense de Electricidad (ICE), han iniciado un proyecto de investigación que analiza las causas y efectos de un posible cambio climático en Costa Rica.
Como primera zona de estudio se escogió la cuenca del río Poás, porque cuenta con datos de precipitación y temperatura en un período de casi 24 años, de caudales en un período de 40 años y se encuentra en el Valle Central, el cual se estima es una de las zona que sería afectada por un posible cambio climático con una reducción de la precipitación o un aumento de la temperatura (MINAE, 2000).

Esta cuenca se ubica en el Valle Central (Fig. 1). Comprende parte de las zonas de recarga de los acuíferos Barba y Colima que están siendo explotados para el abastecimiento de agua potable para gran parte de la población de la Gran Área Metropolitana. La cuenca presenta una forma elipsoidal, drenada por los ríos Tacares, Prendas, Tambor e Itiquís. Se asientan las localidades de San Pedro, Sabanilla y Cinco Esquinas del cantón de Barva. Limita al noreste con el volcán Barva y al norte con el volcán Poás, por lo que la geología está caracterizada por materiales volcánicos, a partir de los cuales se han formado suelos de espesor variable. En las partes baja y media de la cuenca son frecuentes los cultivos de café y caña, mientras que las partes altas están dedicadas a los pastos y algunos parches de bosques secundarios.

Los objetivos específicos de este trabajo comprenden cuantificar la distribución del recurso hídrico por medio de balances hídricos considerando un período de casi 40 años que incluyen el fenómeno del Niño y la Niña. Además, se pretende demostrar la validez de los balances hídricos en la cuantificación del recurso hídrico por medio de la comparación de los resultados de estos con datos de aforos. Finalmente, se busca demostrar por medio de información estratigráfica y paleontológica la ocurrencia de cambios climáticos en el pasado, que han modificado las condiciones de precipitación y temperatura promedio, lo cual debe tenerse en cuenta a la hora de evaluar la influencia de las actividades antrópicas sobre la variabilidad climática.

En cuanto a la información base requerida para el análisis, se solicitaron datos de precipitación y temperatura al Instituto Meteorológico Nacional (IMN) y se incorporó información sobre aforos en el río Poás obtenida del ICE. Se 


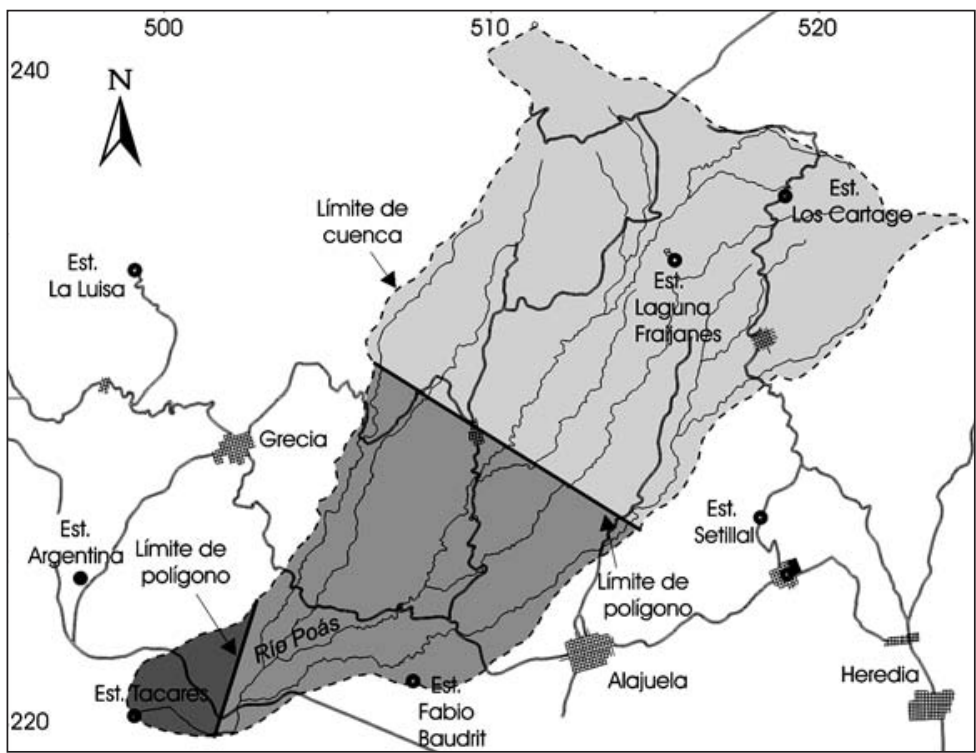

Fig. 1: Mapa de ubicación de las zonas de estudio.

utilizaron los datos de precipitación y temperatura de varias estaciones meteorológicas ubicadas en la cuenca del río Poás y administradas por el IMN en el catastro de las series de precipitaciones medidas en Costa Rica. Con los datos de precipitación, temperatura y capacidad de infiltración se han elaborado balances hídricos, calibrados con datos de caudales.

\section{LA INFLUENCIA DEL FENÓMENO EL NIÑO-LA NIÑA, OSCILACIÓN DEL SUR SOBRE LA DISTRIBUCIÓN DEL RECURSO HÍDRICO EN LA CUENCA DEL RÍO POÁS}

La simulación de las condiciones climáticas que se presentarían con un posible cambio climático para el caso de Costa Rica incluyen una disminución de la precipitación en algunas regiones, con una probable variación (disminución ?) de la recarga que reciben los acuíferos. Una de las zonas donde ocurriría una disminución de la precipitación sería la península de Nicoya y en menor proporción en el Valle Central (MINAE,
2000). Esto traería consecuencias sobre la distribución de la recarga a los acuíferos y en la escorrentía superficial.

Según algunos investigadores las actividades del ser humano tienen implicaciones sobre el cambio climático caracterizado por un aumento de la temperatura. Cuando se introducen en un modelo climático los factores de forzamiento natural más importantes, como por ejemplo la radiación solar y las erupciones volcánicas, el modelo no es capaz de reproducir la tendencia de fuerte calentamiento detectado en las últimas décadas. Por otro lado, cuando se introduce en el modelo el forzamiento debido a los gases antropogénicos de efecto invernadero, los resultados se asemejan mucho mejor a los datos reales observados. Así mismo, cuando se introduce en el modelo tanto los efectos naturales como los de la acción humana, el modelo se ajusta bastante bien a las tendencias observadas en la temperatura superficial del planeta. Esto significa que el cambio climático registrado durante los últimos 40 años del siglo XX se pueden atribuir al calentamiento atmosférico producido, en parte, por los gases de efecto invernadero. 


\section{El fenómeno del Niño-La Niña, y ENOS}

El Niño es un fenómeno climático cíclico que tiene un origen natural. Se caracteriza entre otros elementos oceanográficos y atmosféricos, por un calentamiento intenso y anormal de las aguas superficiales en el océano Pacífico ecuatorial frente a las costas de Perú y Ecuador. Otro aspecto que en ocasiones se presenta es una alteración de la presión atmosférica, lo que provoca un cambio en la dirección y velocidad del viento sobre la misma región. A esta alteración se le conoce con el nombre de Oscilación del Sur (OS). Cuando estos dos fenómenos se presentan al mismo tiempo se le llama ENOS (El Niño/Oscilación del Sur). Estudios recientes asocian una mayor frecuencia e intensidad de dicho evento con el efecto invernadero y el cambio climático (DIRDN, 1998; Suplee, 1999). Durante las fases del Niño las temperaturas aumentan por encima de lo normal, mientras que en épocas de la Niña se encuentran por debajo de lo normal.

Se han identificado varios eventos de sequías asociados al Niño en la primera mitad del siglo XX, como por ejemplo en 1925 y 1941 (Brenes, 1988). No obstante, en la segunda mitad del siglo los eventos fueron más frecuentes

Cuadro 1

Períodos con evidencias del fenómeno El Niño en Costa Rica

\begin{tabular}{lc}
\hline Período & $\begin{array}{c}\text { Duración } \\
\text { (Meses) }\end{array}$ \\
\hline Marzo a Noviembre 1953 & 9 \\
Abril 1957 a Junio 1958 & 15 \\
Junio 1963 a Febrero 1964 & 9 \\
Mayo 1965 a Junio 1966 & 14 \\
Abril 1972 a Marzo 1973 & 12 \\
Agosto 1976 a Marzo 1977 & 8 \\
Julio 1977 a Enero 1978 & 7 \\
Abril 1982 a Julio 1983 & 16 \\
Agosto 1986 a Febrero 1988 & 19 \\
Marzo 1991 a Julio 1992 & 13 \\
Febrero 1993 a Setiembre 1993 & 8 \\
Julio 1994 a Marzo 1995 & 9 \\
Abril 1997 a Abril 1998 & 12 \\
\hline
\end{tabular}

Fuente: Alfaro (2001).
(Cuadro 1). El evento del Niño tiene efectos sobre el Valle Central, con un aumento de la temperatura y una disminución de la precipitación y en el número de días con lluvia en los meses de junio a setiembre (Chacón, 1993).

\section{Distribución del recurso hídrico en la cuenca del río Poás}

Es posible encontrar en la historia hidrometeorológica de la cuenca del río Poás algunas condiciones climáticas (frecuencia de períodos de sequías) que se podrían asociar con un posible cambio climático, debido a la frecuencia de ocurrencia del Niño-La Niña y su intensidad. De hecho de acuerdo a los datos meteorológicos de la cuenca es evidente, que se han presentado varios años de menor precipitación asociados al evento climático el Niño y años con mayor precipitación asociados con la Niña.

Es posible analizar la variación en la distribución del agua durante estos períodos por medio de balances hídricos, que permiten entre otros aspectos estimar la variación de la recarga a los acuíferos y de la escorrentía superficial por medio de datos hidrometeorológicos, principalmente precipitación, temperatura, caudales y características del suelo. Así mismo, es posible cuantificar la variación de los elementos del balance hídrico durante períodos secos y húmedos para utilizarlos en la planificación del recurso hídrico.

Para la cuenca del río Poás es posible utilizar información hidrometeorológica proveniente de las estaciones Fabio Baudrit (84023), La Argentina de Grecia (84003), Los Cartagos y Laguna de Fraijanes (84030). Los períodos de registro de la información meteorológica para estas estaciones varían de manera significativa, no obstante, es posible identificar algunas estaciones con períodos de registro similares.

Para las estaciones Fabio Baudrit y La Argentina de Grecia se ha logrado recopilar la información más completa de precipitación, temperatura mínima y máxima. No obstante, solo es 
posible observar un lapso de tiempo común que va de 1976 a 2001 (Cuadro 2). Estas estaciones se ubican a elevaciones similares en la parte baja de la cuenca. La estación Fabio Baudrit se encuentra a una elevación de $840 \mathrm{~m}$ y la Argentina a $760 \mathrm{~m}$ (Fig. 1). Por su parte, las estaciones Los Cartagos y la Laguna de Fraijanes (1850 m s.n.m) comparten elevaciones muy similares; sin embargo, no se dispone de datos de temperatura para la estación Los Cartagos.

La evapotranspiración potencial se obtuvo por el método de Blaney \& Cridle, utilizando datos de temperaturas máximas y mínimas de las estaciones supra mencionadas. Los datos de caudales se obtuvieron de la estación Tacares, ubicada a una elevación de 598 m, sobre el río Poás, que abarca un área de drenaje de $201,5 \mathrm{~km}^{2}$. El registro de datos comprende el período de 1961 al 2001 y ha permitido realizar la calibración de los balances hídricos (Cuadro 2).

El cálculo de la infiltración se realizó siguiendo la metodología propuesta por Schosinsky \& Losilla (2000), considerando un suelo franco arenoso con una capacidad de campo de $243 \mathrm{~mm}$, un punto de marchitez de $115 \mathrm{~mm}$, una profundidad de raíces de $500 \mathrm{~mm}$ y una capacidad de infiltración de $1860 \mathrm{~mm} /$ día. Por su parte, la recarga a los acuíferos se calculó tomando en cuenta el agua disponible, la humedad del suelo inicial, la humedad del suelo final y el cambio de humedad del suelo. En el cuadro 3 se muestra el esquema de cálculo para cada uno de los factores que intervienen en el balance hídrico, así como el cálculo de la evapotranspiración potencial por medio de Blaney \& Cridle.

Para la calibración de los balances hídricos se compararon los datos de caudal pro- medio anual calculado por el balance hídrico con el caudal promedio anual registrados en la estación Tacares. De los 24 años analizados, en 16 de ellos el porcentaje de diferencia (error) fue de un $\pm 10 \%$ (Cuadro 4). Las mayores diferencias se presentaron en los años 1996, 1997 y 1998, los cuales coincidieron con eventos muy acentuados de la Niña y el Niño. Tanto así que en 1996 se produjo el huracán Cesar, uno de los más destructivos para Costa Rica y en 1998 se produjo el huracán Mitch, que está considerado el más destructivo en la historia de la región centroamericana, afectando a Costa Rica con el aporte de precipitaciones.

La precipitación anual en la cuenca varía de 2000 a $3500 \mathrm{~mm}$ y se distribuye en períodos húmedos y secos (Fig. 2). De 1976 a 1984 y de 1995 al 2001 se aprecian períodos húmedos con valores de precipitación que superan los 2500 mm, mientras que de 1985 a 1994 los valores rondan los $2000 \mathrm{~mm}$. Uno de los valores más bajos registrados se presentó en 1986, debido a la ocurrencia de un evento tipo Niño de moderada intensidad, lo que produjo una estación lluviosa deficitaria (Ramírez, 1992). Con respecto a los caudales aforados en el río Poás, el período seco de 1985 a 1994 se identifica por caudales anuales promedio menores de $10 \mathrm{~m}^{3} / \mathrm{s}$, mientras que para el resto los valores superan el mencionado valor (Fig. 3).

En el año 1986 se registró la menor precipitación en la cuenca durante el período analizado, alcanzando $2019 \mathrm{~mm}$, lo que significó un $22 \%$ menos que el promedio. Mientras tanto, la mayor precipitación se registró en el año de 1996 con $3646 \mathrm{~mm}$, es decir, un $40 \%$ mayor que el promedio. Para el año 1986 se obtuvo una recarga

Cuadro 2

Períodos con registro de datos en la cuenca del río Poás

\begin{tabular}{lcccccc}
\hline Estación & Lat. & Long. & Elevación $(\mathrm{m})$ & Precipitación & Temperatura & Caudal \\
\hline Fabio Baudrit & $10^{\circ} 01$ & $84^{\circ} 16$ & 840 & $1968-1986$ & $1961-2001$ & -- \\
La Argentina & $10^{\circ} 02$ & $84^{\circ} 21$ & 760 & $1968-1986$ & $1970-2001$ & -- \\
Los Cartagos & $10^{\circ} 08$ & $84^{\circ} 10$ & -- & $1968-1986$ & -- & -- \\
Lag. Fraijanes & $10^{\circ} 09$ & $84^{\circ} 12$ & 1850 & $1976-2000$ & $1976-2001$ & -- \\
Tacares & $10^{\circ} 01$ & $84^{\circ} 20$ & -- & -- & -- & $1968-1986$ \\
\hline
\end{tabular}


Cuadro 3

Balance hídrico de suelos para la cuenca del río Poás en el año 1985

\begin{tabular}{|c|c|c|c|c|c|c|c|c|c|c|c|c|c|}
\hline Mes & Ene. & Feb. & Mar. & Abr. & May. & Jun. & Jul. & Ago. & Set. & Oct. & Nov. & Dic. & TOTAL \\
\hline $\mathrm{P}(\mathrm{mm})$ & 24,6 & 31,7 & 2,1 & 67,1 & 197,2 & 300,9 & 267,4 & 240,5 & 317,9 & 363,7 & 201,3 & 46,29 & 2060,1 \\
\hline $\mathrm{Pe}(\mathrm{C}<5)(\mathrm{mm})$ & 24,6 & 31,7 & 0,0 & 67,1 & 197,2 & 300,9 & 267,4 & 240,5 & 317,9 & 363,7 & 201,3 & 46,29 & 2058,1 \\
\hline $\mathrm{I}(\mathrm{mm})$ & 1,2 & 1,6 & 0,1 & 3,4 & 9,9 & 15,0 & 13,4 & 12,0 & 15,9 & 18,9 & 10,1 & 2,3 & 103,0 \\
\hline $\mathrm{P}-$ Int.(mm) & 23,4 & 30,16 & 1,95 & 63,8 & 187,3 & 285,2 & 254,1 & 228,5 & 302,1 & 345,5 & 191,2 & 44,0 & 1957,1 \\
\hline $\mathrm{Pi}(\mathrm{mm})$ & 21,7 & 27,9 & 1,8 & 59,1 & 173,5 & 264,2 & 235,3 & 211,6 & 279,8 & 320,1 & 177,1 & 40,7 & 1812,9 \\
\hline $\mathrm{Pe}(\mathrm{mm})$ & 1,7 & 2,2 & 0,14 & 4,7 & 13,8 & 21,0 & 18,7 & 16,8 & 22,3 & 25,5 & 14,1 & 3,2 & 144,2 \\
\hline $\mathrm{Pe}(\mathrm{m} 3 / \mathrm{ha})$ & 17,2 & 22,2 & 1,44 & 47,0 & 138,1 & 210,1 & 187,2 & 168,4 & 222,6 & 254,6 & 140,9 & 32,4 & 1442,1 \\
\hline $\operatorname{ETP}(\mathrm{mm})$ & 83,1 & 78,7 & 91,27 & 94,1 & 96,7 & 97,3 & 97,0 & 93,8 & 88,3 & 89,6 & 84,3 & 85,5 & 1079,8 \\
\hline P-ETP & 0,0 & 0,0 & 0,00 & 0,0 & 100,5 & 202,9 & 170,8 & 146,7 & 229,7 & 274,2 & 117,0 & 0,0 & 1241,3 \\
\hline $\operatorname{AgD}(\mathrm{mm})$ & $-61,4$ & $-50,8$ & $-89,5$ & $-35,0$ & 76,9 & 166,9 & 138,3 & 117,8 & 191,5 & 230,5 & 92,8 & $-44,7$ & 733,1 \\
\hline $\mathrm{HSi}(\mathrm{mm})$ & 83,5 & 22,0 & 0,0 & 0,0 & 0,0 & 76,9 & 128,2 & 128,2 & 128,3 & 128,3 & 128,3 & 128,2 & 951,9 \\
\hline HSf (mm) & 22,0 & 0,0 & 0,0 & 0,0 & 76,9 & 128,2 & 128,2 & 128,2 & 128,3 & 128,2 & 128,2 & 83,5 & 951,9 \\
\hline CHs (mm) & $-61,5$ & $-22,0$ & 0,0 & 0,0 & 76,9 & 51,4 & 0,0 & 0,0 & 0,0 & 0,0 & 0,0 & $-44,7$ & 0,0 \\
\hline $\mathrm{DCC}(\mathrm{mm})$ & 106,2 & 128,2 & 128,2 & 128,2 & 51,4 & 0,0 & 0,0 & 0,0 & 0,0 & 0,0 & 0,0 & 44,7 & 587,1 \\
\hline $\mathrm{RP}(\mathrm{mm})$ & 0,0 & 0,0 & 0,0 & 0,0 & 0,0 & 115,5 & 138,3 & 117,8 & 191,5 & 230,5 & 92,8 & 0,0 & 886,4 \\
\hline $\mathrm{RP}(\mathrm{m} 3 / \mathrm{Ha})$ & 0,0 & 0,0 & 0,0 & 0,0 & 0,0 & 1155,2 & 1382,8 & 1178,1 & 1915,1 & 2305,0 & 928,1 & 0,0 & 8864,3 \\
\hline ETR $(\mathrm{mm})$ & 83,1 & 50,0 & 1,8 & 59,1 & 96,7 & 97,3 & 97,0 & 93,8 & 88,3 & 89,6 & 84,3 & 85,5 & 926,5 \\
\hline $\mathrm{NR}(\mathrm{mm})$ & 106,2 & 128,2 & 128,2 & 128,2 & 51,4 & 0,0 & 0,0 & 0,0 & 0,0 & 0,0 & 0,0 & 44,7 & 587,1 \\
\hline $\mathrm{T}\left({ }^{\circ} \mathrm{C}\right)$ & 18,6 & 19,08 & 19,4 & 20,1 & 20,0 & 20,1 & 19,6 & 19,4 & 19,3 & 19,3 & 19,2 & 19,1 & \\
\hline $\mathrm{Kt}$ & 0,8 & 0,84 & 0,9 & 0,9 & 0,9 & 0,9 & 0,9 & 0,84 & 0,8 & 0,8 & 0,8 & 0,8 & \\
\hline $\mathrm{Kc}$ & 0,8 & 0,75 & 0,7 & 0,7 & 0,7 & 0,7 & 0,7 & 0,7 & 0,7 & 0,7 & 0,7 & 0,7 & \\
\hline $\mathrm{P}(\%)$ & 8,1 & 7,5 & 8,45 & 8,4 & 8,6 & 8,6 & 8,9 & 8,7 & 8,2 & 8,3 & 7,9 & 8,1 & \\
\hline $\operatorname{ETP}(\mathrm{mm})$ & 83,1 & 78,8 & 91,27 & 94,1 & 96,7 & 97,3 & 97,0 & 93,8 & 88,3 & 89,6 & 84,3 & 85,5 & \\
\hline
\end{tabular}

P: precipitación; Pe: precipitación efectiva $(\mathrm{mm})$; Pi: precipitación infiltrada $(\mathrm{mm})$; Pe: precipitación que escurre $(\mathrm{mm})$; $\mathrm{I}$ : Intercepción (mm), ETP: evapotranspiración potencial; AgD: agua disponible; Hsi: humedad del suelo inicial; HSf: humedad del suelo final; CHs: cambio de humedad del suelo; DCC: déficit de capacidad de campo; RP: recarga potencial; ETR: evapotranspiración real; NR: necesidad de riego.

\section{Cuadro 4}

Caudales (Q) medios y diferencia porcentual expresado como lámina de agua

\begin{tabular}{|c|c|c|c|}
\hline AÑO & Q. medido $(\mathrm{mm})$ & Q. calculado (mm) & Diferencia $(\%)$ \\
\hline 1976 & 130,11 & 117,01 & 10,07 \\
\hline 1977 & 103,08 & 118,90 & $-15,34$ \\
\hline 1978 & 125,76 & 126,17 & $-0,33$ \\
\hline 1979 & 153,53 & 152,66 & 0,57 \\
\hline 1980 & 156,05 & 148,16 & 5,32 \\
\hline 1981 & 155,95 & 154,00 & 1,25 \\
\hline 1982 & 143,76 & 133,80 & 6,93 \\
\hline 1983 & 145,41 & 138,68 & 4,62 \\
\hline 1984 & 168,23 & 166,88 & 0,80 \\
\hline 1985 & 122,57 & 103,44 & 15,61 \\
\hline 1986 & 96,40 & 109,71 & $-13,81$ \\
\hline 1990 & 140,75 & 141,89 & $-0,81$ \\
\hline 1991 & 123,12 & 120,40 & 2,21 \\
\hline 1992 & 117,64 & 120,79 & $-2,68$ \\
\hline 1993 & 141,25 & 131,29 & 7,05 \\
\hline 1994 & 95,56 & 111,49 & $-16,66$ \\
\hline 1995 & 179,41 & 184,45 & $-2,81$ \\
\hline 1996 & 184,31 & 226,45 & $-22,87$ \\
\hline 1997 & 121,65 & 152,75 & $-25,56$ \\
\hline 1998 & 159,68 & 207,54 & $-29,97$ \\
\hline 1999 & 189,07 & 208,82 & $-10,45$ \\
\hline 2000 & 146,86 & 144,26 & 1,77 \\
\hline 2001 & 145,49 & 161,28 & $-10,86$ \\
\hline
\end{tabular}




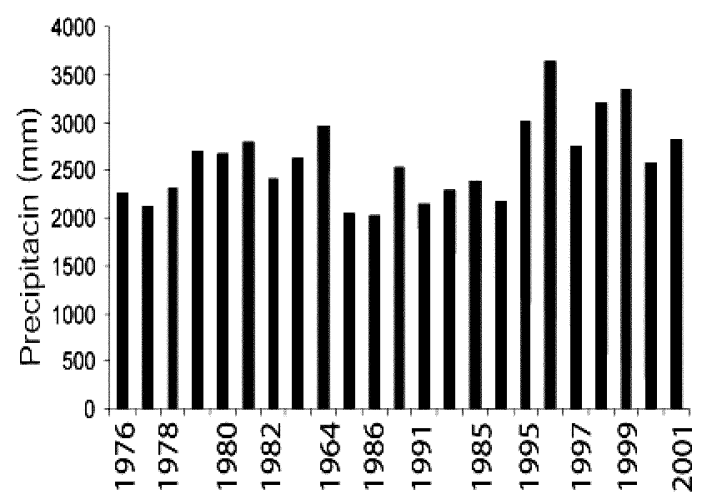

Fig. 2: Precipitación total anual en la cuenca del río Poás.

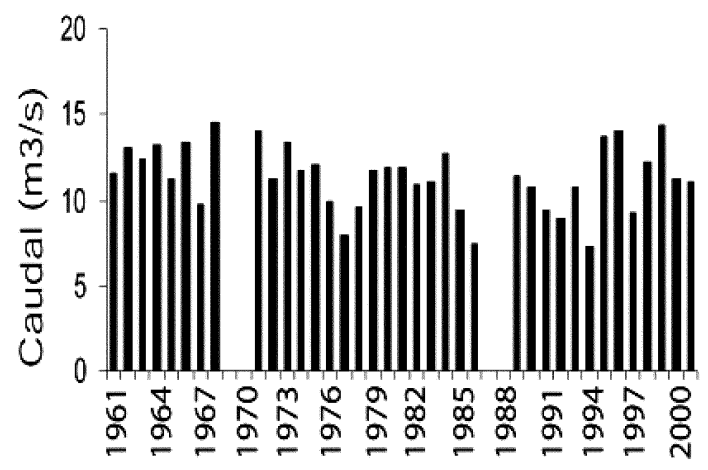

Fig. 3: Variación anual del caudal registrada en la estación Tacares.

potencial de $850 \mathrm{~mm}$, un $36 \%$ menor que el promedio y para 1996 se obtuvo una recarga de $2205 \mathrm{~mm}$, es decir, un $65 \%$ mayor que el promedio. Además, la escorrentía fue un $32 \%$ menor en 1986 y un $30 \%$ mayor en 1996 que el promedio respectivamente.

El porcentaje de la precipitación que se considera como recarga potencial media mensual varía de 24\% (año 1986) hasta un $48 \%$ (1996). La recarga total anual varía de $850 \mathrm{~mm}$ hasta $2205 \mathrm{~mm}$ y la mayor parte de la recarga ocurre en los meses de setiembre y octubre

La distribución de la recarga está caracterizada por períodos alternos bien definidos donde se observa una recarga anual mayor o menor dependiendo de la ocurrencia de eventos Niño o Niña (Fig. 4). Por ejemplo, el período 1976-1984 se caracteriza por una recarga anual que supera los 1000 mm, mientras que de 1985 hasta 1994 la

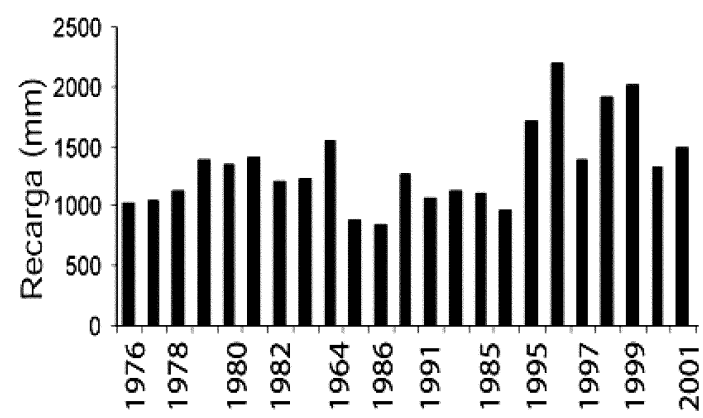

Fig. 4: Recarga total anual en la cuenca del río Poás.

recarga disminuye y de 1995 al 2001 aumenta nuevamente. Los períodos más secos se asocian a la ocurrencia de los eventos Niño; por el contrario, los períodos más húmedos se asocian con la mayor frecuencia de la Niña.

\section{EVIDENCIAS PALEONTOLÓGICAS Y ESTRATIGRÁFICAS DE CAMBIOS CLIMÁTICOS EN COSTA RICA}

Costa Rica, con una evolución geológica relativamente reciente (Jurásico Inferior, aproximadamente $200 \mathrm{Ma}$ ) y sin variaciones latitudinales tan grandes en el transcurso de su desarrollo, como para experimentar cambios climáticos extremos, presenta evidencias en el registro sedimentario (rocas y fósiles), de haber estado sometida a condiciones climáticas variables. Los fósiles más antiguos conocidos son los radiolarios, los cuales se encuentran en grandes cantidades conformando rocas (radiolaritas). Esto es una evidencia de que en la época en que se depositaron (hace $190 \mathrm{Ma}$ ), prevalecían en el área que actualmente ocupa nuestro país, condiciones de temperatura media más alta que las actuales.

Posteriormente, al final del período Cretácico (hace unos $70 \mathrm{Ma}$ ), las condiciones paleogeográficas indican que existían áreas emergidas con plataformas someras que permitieron el desarrollo de formaciones carbonatadas y arrecifes, los cuales requieren para su desarrollo de condiciones ecológicas muy específicas, que sólo se dan en mares tropicales o subtropicales, como son temperaturas entre $\operatorname{los} 18^{\circ} \mathrm{y}$ 
$\operatorname{los} 23^{\circ} \mathrm{C}$, aguas limpias, turbulentas pero $\sin$ turbidez y buena iluminación.

El Terciario Inferior no presenta elementos diagnósticos que permitan interpretar cambios en el clima, aunque se menciona un enfriamiento general (Gómez, 1986). Sin embargo, durante el Eoceno Medio- Superior, Oligoceno Superior $(50-25 \mathrm{Ma})$, el desarrollo de plataformas carbonatadas en diversas partes del país, es un buen indicador de un clima tropical bien establecido. El Terciario Superior inicia con un Mioceno cálido, posiblemente muy lluvioso, sobretodo al final, cuando abundan los bosques, que actualmente podemos reconstruir en los diferentes yacimientos de carbón y en la floras fósiles encontradas. Al final del Plioceno y durante el Pleistoceno, existen diferentes evidencias de que el enfriamiento que afectó el Hemisferio Norte también incidió en una gran parte de nuestro país. Existen claras evidencias de la existencia de plantas típicas de climas más fríos en las partes altas del país (cordilleras y principalmente las faldas de los volcanes sin actividad), como por ejemplo Quercus sp., Pinus sp. y Podocarpus sp. (Gómez, 1986). La megafauna, típica de clima fríos, proveniente de Norte América, aprovechó estos cambios para distribuirse y migrar hacia latitudes más bajas e incluso hacia Sur América.

Según Gómez (1986), el Valle Central era como es hoy, más seco hacia el oeste, pero también mucho más frío, mientras que en la parte Oriental, abundaban las fuentes de aguas termales, por lo que el autor propone que en conjunto con los depósitos volcánicos del Noreste y Este, haya constituido un refugio con clima más cálido para muchas especies de plantas y animales de origen suramericano y tropicales. Las floras fósiles evidencian un clima con temperatura moderada y con una humedad relativamente alta.

Son abundantes también las evidencias de hielo en las partes más altas de la cordillera de Talamanca, tanto morfológicas, lagos y depósitos asociados a glaciares (Protti, 1996; Jiménez, 1997), como florísticas, la vegetación de tipo páramo. Existen, además, evidencias de la precencia de vegetación que actualmente vive sobre los $1200 \mathrm{~m}$ en zonas que hoy sólo tienen $800 \mathrm{~m}$ de elevación, como, por ejemplo, las localidades de
Hone Creek y Fila del Carbón con Quercus y Podocarpus (Gómez, 1986) y La Palmera de San Carlos, con Quercus (Pérez, 1998).

Otra evidencia importante son los abundantes depósitos lacustres con diatomeas que se encuentran en varias localidades de Costa Rica durante un período de bajas temperaturas y de relativa calma de la actividad volcánica acaecida entre los 3 a 1,6 Ma (Alvarado et al., 1993). En una época más reciente, se encuentran evidencias en nuestro país de la Pequeña Edad del Hielo, como puede deducirse de las crónicas de algunos conquistadores, hace 500 años (Jiménez, 1997) y del crecimiento de las colonias de corales (Jiménez, C, com. oral).

\section{CONCLUSIONES}

Como se ha comprobado con la evidencia sedimentaria y paleontológica la ocurrencia de cambios climáticos en la historia de Costa Rica es un proceso natural en el cual el hombre no ha tenido intervención alguna. El aumento de temperatura media detectado en los últimos años y la ocurrencia de los eventos meteorológicos denominados Niño o Niña no se pueden relacionar con la intervención directa del hombre pues no hay elementos que indiquen que no se trata de un proceso natural.

A partir de la información suministrada por los balances hídricos de suelos implementados en años de períodos secos y húmedos es posible visualizar la reducción de la recarga con respecto a los períodos normales o muy húmedos. La calibración de los balances con los valores de caudales medidos en la mayoría de casos y en condiciones normales se ha realizado dentro de un margen de error menor al 10\%.

En los años 1985 y 1986 se calcularon los valores de recarga más bajos, 850 y $886 \mathrm{~mm}$ respectivamente, lo cual implica una reducción con respecto al promedio de un $30 \%$. No es posible asegurar si un posible cambio climático aumentaría la frecuencia de estos casos. No obstante, es probable que se presenten en el futuro eventos de sequía para los cuales deberían establecerse planes de mitigación. 


\section{RECOMENDACIONES}

Un inminente cambio climático implicaría la reducción de la disponibilidad del agua en ciertas zonas, por lo que se requiere un mejor control sobre el uso y consumo de agua durante las épocas de estiaje. No es recomendable que agua de excelente calidad se utilice para labores de limpieza en ciertos sectores urbanos, mientras que en otros se da el racionamiento por déficit de agua. Además, se debe atacar el despilfarro del agua, por medio de tarifas debidamente establecidas, así como establecer incentivos para ahorrar el agua. Sin embargo, es necesario ir propiciando esta conciencia con una campaña que se inicie en coordinación con las municipalidades, las instituciones encargadas de la protección de los recursos naturales y las comunidades.

\section{AGRADECIMIENTOS}

El apoyo de varias instituciones y personas permitió la realización de éste informe en un tiempo muy corto. El apoyo económico del Programa de Naciones Unidas para el desarrollo (PNUD) fue fundamental. Se agradece la colaboración del IMN, por el suministro de los datos básicos. Así como el apoyo de Guillermo Alvarado y Rafael Chacón del ICE.

Esta publicación es el resultado de parte del proyecto 113-A2-519 de la Vicerrectoría de Investigación (UCR).

\section{REFERENCIAS}

ALVARADO, G., KUSSMAUL, S., CHIESA, S., GILLOT, P., APPEL, H. WÖRNER, G \& RUNDLE, C. 1993. Resumen cronoestratigráfico de las rocas ígneas de Costa Rica basado en dataciones radiométricas. South American Earth Sci. 6 (3): 151-168.

ALFARO, E., 2001: Variabilidad del clima regional. - 44 págs. Comité Regional de Recursos Hídricos. Curso preparándonos para enfrentar la variabilidad climática. San José.

BRENES, C., 1988: El domo térmico de Costa Rica durante el fenómeno de El Niño 1972-1973. - Ciencia y Tecnología 12 (1-2): 53-58.
CHACÓN, R., 1993: Aspectos de la influencia del fenómeno de El Niño en el clima costarricense. - Rev. Geog. América Central, 27: 53-66.

DIRDN, 1998: Qué es el Niño. - DIRDN Informa No. 12. 711. San José.

ECLAC, 1993: Climate change and water management in Latin America and the Caribbean.-106 págs. División de Recursos Naturales y Energía. [Sin ciudad de publicación].

GÓMEZ, W., 1986: Vegetación y clima de Costa Rica. - 327 págs. Edit. UNED, San José

JIMÉNEZ, I., 1997: Pequeña edad del hielo cuando Colón llegó aquí. - En Eco Católico, 3455:4.

MINAE, 2000: Primera comunicación nacional ante la Convención Marco de las Naciones Unidas sobre cambio climático. - 65 págs. IMN, San José.

OBSERVATORIO DEL DESARROLLO, 2002: Geo Costa Rica: una perspectiva sobre el medio ambiente. -161 págs. MINAE, PNUMA. San José.

PÉREZ, A. 1998. Evaluación paleo-ambiental de una localidad con flora fósil del Pleistoceno, La Palmera de San Carlos Provincia de Alajuela. - 60 págs.[Inf. Final práctica dirigida para optar al título de Bachiller en Manejo de Recursos Naturales.] UNED.

PNUMA \& WMO, 2002: Cambio climático y desarrollo. 465 págs. Gómez, L. (ed.). Yale School of Forestry, San José.

PROTTI, R., 1996. Evidencias de glaciación en el Valle del General (Costa Rica) durante el Pleistoceno Tardío. - Rev. Geol. América Central 19/20: 75-85.

RAMÍREZ, P., 1992: Descripción de situaciones climatológicas que pueden producir desastres en Costa Rica. - Rev. Geog. América Central. 25/26. I y II semestre: 153-169.

SCHOSINKY, G. \& LOSILLA, M., 2000: Modelo analítico para determinar la infiltración con base en la lluvia mensual. - Rev. Geol. América Central, 23: 43-55.

SUPLEE, C., 1999: El Niño, La Niña: Natures vicious cycle. - National Geographic, 195( 3): 75- 95.

ZÁRATE, E., 2001: Eventos meteorológicos extremos que afectan a Centroamérica. - 18 págs. Comité Regional de Recursos Hídricos. Curso preparándonos para la variabilidad climática. San José. 